\title{
LA CIUDAD SUBDESARROLLADA *
}

\author{
VÍCTOR L. URQUIDI \\ El Colegio de México
}

Las ciudades contemporáneas pertenecen a los pobres.

J. F. C. TuRner, en el Seminario Interregional de las Naciones Unidas sobre La Política y la Planeación del Desarrollo Urbano, Pittsburgh, 1966.

El desmoronamiento de Roma fue el resultado final de su crecimiento exagerado... ejemplo amenazador de la expansión incontrolada, la explotación sin escrúpulos y el exceso materialista.

L.EWIS MUMFord, The City in History, 1961.

Contener el problema evitando así que empeore.

De un informe del Banco Interamericano de Desarrollo, 1965.

\section{I}

El CRECIMIENTo uRBANo, que se registra a una tasa bastante más elevada que la del aumento general de la población, está ganando importancia rápidamente en la lista de los problemas económicos y sociales no resueltos, y tal vez insolubles, de los países del mundo menos desarrollado. Aunque en algunas de estas naciones hubo indicaciones claras de urbanización acelerada durante los años cuarenta, sobre todo bajo el impacto de la segunda guerra mundial y sus consecuencias, sólo a partir de 1950, aproximadamente, es cuando el proceso ha adquirido velocidad. Sin embargo, este proceso es todavía de crecimiento urbano más que de desarrollo urbano, si por desarrollo se entiende un proceso de cambio orgánico en función de objetivos y que pueda contribuir a un avance económico sólido y a un mayor bienestar sin que se creen desequilibrios penosos o se produzcan nuevas formas de malestar social. Las ciudades, de condiciones y tamaños distintos, están creciendo: contienen a más habitantes, se extienden de manera horizontal y vertical, concentran en su medio más actividad comercial e industrial, atraen a la mayor parte de las inversiones y los servicios, y se benefician proporcionalmente más que las áreas rurales del avance educativo y de la cultura en general. Pero el crecimiento

* Trabajo presentado a un simposio sobre El hombre en La Ciudad del Futuro llevado a cabo en Río Piedras, Puerto Rico, en octubre de 1967, bajo los auspicios de Lions International y la Universidad de Puerto Rico. El original se publicó en inglés en la obra colectiva de Richard Eells y Clarence Walton, Man in the City of the Future, Nueva York, Macmillan-Arkville Press, 1968, pp. 73-97. La traducción es de Joaquín Urquidi. 
de las ciudades en los países más pobres, no previsto en su escala actual, ha traído una nueva dimensión al desarrollo económico y social -el requisito de que el crecimiento económico, difícil en sí mismo, de alguna manera se haga congruente con las normas apetecibles de la vida urbana y tome en cuenta las varias y complejas fuerzas que configuran a la ciudad y a la conducta de sus habitantes.

Los problemas de la urbanización son, claro está, mundiales. La ciudad es una preocupación principal de los sociólogos, los planificadores, los estadistas y los políticos; de los medios de comunicación masiva, y de la gente común de las naciones del mundo industrialmente desarrollado. Aunque las descripciones espeluznantes de las ciudades industriales del siglo xIX son principalmente cuestión de historia, la riqueza económica actual de las naciones más avanzadas no ha conducido a soluciones satisfactorias desde el punto de vista social. 1 Entre las condiciones de vida y de vivienda de las familias en los niveles de ingresos superiores y las de los estratos inferiores persisten contrastes casi sin medida. Una apreciación reciente y repentina de esas diferencias está requiriendo una concienzuda reevaluâción de las políticas de desarrollo urbano, y la consideración no sólo de los problemas internos de cada ciudad, sino también de las interrelaciones entre los centros urbanos y del significado último de la urbanización para la nación entera.

La gravedad de estos problemas en los países desarrollados del mundo occidental, y las implicaciones casi aterradoras de los futuros conurbios megalopolitanos que se prevén, no se pueden subestimar. Sin embargo, la mayor parte de la literatura escrita sobre esta materia, ya sea pesimista o utópica, trata a la ciudad, o a la cadena de ciudades, como accesible a algún tipo de esfuerzo concertado por parte de los planificadores y las autoridades a niveles locales y nacionales: los recursos, en potencia, están disponibles y lo que se necesita, en general, es resolución en función de prioridad social y política (aparte de ciertas controversias entre las diferentes escuelas de planificación urbana). En cambio, en las partes menos desarrolladas del mundo -donde, no debe olvidarse, el ingreso medio por habitante varía, en diferentes regiones, de una veinteava a una cuarta parte de los ingresos medios en los países industrialmente avanzados- el problema estriba en una virtual indisponibilidad de recursos, además del aspecto, que dista de ser simple, de la toma de decisiones (en relación con lo cual las perspectivas pueden ser incomparablemente menos alentadoras). La urbanización en los países menos desarrollados es necesaria para el desarrollo moderno, pero está exhibiendo algunas de las peores características del crecimiento de las ciudades en las naciones industriales, a lo que se agrega el cambio social sin precedente que resulta de las tasas elevadas de crecimiento demográfico y de los movimientos en masa de población de las áreas rurales a las urbanas.

Todas estas circunstancias están produciendo la ciudad subdesarrollada - la ciudad de las naciones subdesarrolladas. Debido a las situaciones económicas y políticas en que se desenvuelve, este tipo de ciudad bien puede llegar a convertirse en la ciudad subdesarrollada permanente. A continuación se intenta examinar las condiciones ge- 
nerales que rodean esta faceta particular del "Decenio del Desarrollo" $\mathrm{y}$ algunos de los lineamientos que podrían servir cuando menos para contener el problema. En la mayor parte, he consultado la literatura reciente sobre el cambio demográfico y el desarrollo urbano. ${ }^{1}$ No pretendo hacer uso de conocimientos especializados - lo cual puede ser una ventaja - sino que trato de ver el problema de manera más general, tal vez como lo haría un economista del desarrollo, aunque no hasta el punto de permitir que el cuadro total se oscurezca y se disuelva en lugares comunes. De manera inevitable, emplearé primordialmente los datos y la experiencia de América Latina, pero gran parte de lo que resulta de este cuadro probablemente se pueda aplicar al crecimiento urbano en Asia y en partes de Africa.

\section{II}

Más de 2200 millones de personas habitan el mundo subdesarrollado, contra cerca de 1000 millones en los países más afortunados en el avance económico. Una medida común del nivel de urbanización es el porciento de la población que vive en localidades de más de 20000 habitantes. En 1920 esta proporción, para el mundo entero, fue del $14 \%$; en $1940,19 \%$, y en $1960,25 \%$; o sea un total de 753 millones de habitantes en 1960.2 De esta cantidad, más de la mitad, unos 380 millones, habitaban las áreas menos desarrolladas: Asia meridional y oriental, América Latina y Africa. ${ }^{3}$ En América Latina, la proporción, en 1960, de los habitantes en localidades de más de 20000 habitantes fue de $32 \%$, mientras que en Asia meridional fue de sólo $14 \%$; en América del Norte, fue de $57 \%$; en Europa, 41, y en la Unión Soviética, 36. Entre 1940 y 1960 el número de habitantes en dichas localidades se duplicó con creces en las áreas menos desarrolladas: en América Latina aumentó en un $170 \%$; en Africa, $164 \%$; en Asia me-

1 Entre las fuentes consultadas particularmente valiosas, se pueden mencionar las siguientes: trabajos presentados por la División de Población de las Naciones Unidas, otros organismos relacionados y expertos individuales al Seminario Interregional de las Naciones Unidas sobre Planeación y Política del Desarrollo Urbano, en Pittsburgh, octubre-noviembre de 1966; escritos presentados a la Conferencia Mundial de Población, Belgrado, septiembre de 1965; Luis Lander y Julio César Funes, "Urbanismo y Desarrollo", en Hacia una politica de integración para el desarrollo de América Latina, Actas del Sexto Congreso Interamericano de Planificación, Caracas, noviembre 6-11, 1966 (San Juan, Puerto Rico, Sociedad Interamericana de Planificación, 1967), pp. 60-110; y Rubén D. Utria, "Los factores estructurales del desarrollo y el problema de la vivienda en América Latina", Boletín Económico de América Latina, Vol. XI, Núm. 2, octubre de 1966, Naciones Unidas, CEPAL, pp. $246-275$.

2 División de Población de las Naciones Unidas, Trends in World Urbanization, 1920-1960, trabajo presentado al Seminario Interregional que se menciona en la nota 1 ; estimación derivada del cuadro 2.

3 Una definición aparentemente más rigurosa de "áreas menos desarrolladas" da un total de sólo 321 millones en este grupo en 1960, o sea el $42.4 \%$ del total. Este agrupamiento supuestamente excluye a Japón, donde el grado de industrialización y de urbanización es mucho más alto y la tasa de incremento demográfico es mucho más baja que en la mayor parte de los países de Asia, aunque el ingreso per capita está por debajo de los niveles occidentales. Sin embargo, no está disponible la subdivisión por regiones de los 321 millones por lo que en el texto se utilizó la cifra más alta. Cf. ibid., cuadro 11.

4. Ibid., cuadro 4. 
ridional, 130 y en Asia oriental, $96 \% .^{5}$ En las áreas desarrolladas, los aumentos variaron del 24 al $76 \%$.

Sin embargo, las localidades que contienen de 20000 a 100000 habitantes no pueden ser tomadas como ciudades en el sentido usual de la palabra. Si se toma como indicadores de la urbanización a las ciudades de más de 100000 habitantes, el porciento de la población mundial que habita en ellas ascendió de 8.6 en 1920 a 12.6 en 1940 y a 17.5 en 1960 ; de 1940 a 1960 hubo un aumento de $82 \% .^{6}$ Hoy en día cerca de 600 millones de personas viven en ciudades de semejante tamaño, y tal vez la mitad se encuentre en los países menos desarrollados, donde las tasas de incremento son, por lo general, más altas.

Aun otro punto conveniente de separación es el límite de 500000 , que distingue a las ciudades grandes de las simples ciudades y localidades urbanas. Para 1960, casi el $12 \%$ de la población mundial, o 352 millones de habitantes, vivía en dichas ciudades grandes, contra un $5 \%$ en 1920. En Asia meridional, América Latina y África, entre los años de 1940 y 1960 , el número de habitantes en ciudades de este rango se triplicó con creces y aumentó casi a esa tasa en Asia oriental; en América del Norte y la Unión Soviética se duplicó y en Europa ascendió sólo una quinta parte. En particular, el decenio de los cincuenta presenció una expansión más rápida de las ciudades grandes y las metrópolis que de las ciudades menores. La concentración urbana aumentó, sobre todo en la Unión Soviética y en las áreas menos desarrolladas. En estas últimas, en 1960, el $46 \%$ de la población urbana vivía ya en ciudades de 500000 o más personas. La rapidez con qụe esto ocurrió en esas regiones no significa, desde luego, que se estaban alcanzando los niveles de urbanización de los países industriales, pero sí es significativo que casi la misma cantidad de personas vivía en ciudades de más de 500000 habitantes en los países más pobres que en los más ricos. ${ }^{7}$ Con sólo considerar a América Latina, se encuentra que el $17 \%$ de su población total habitaba este tipo de ciudad, una proporción más elevada que la de otras regiones menos desarrolladas, igual a la de Europa, y superior a la media mundial. ${ }^{8}$

Dadas las tasas diferenciales de incremento de la población entre las áreas desarrolladas y las subdesarrolladas, se espera que para el año 2000 éstas (excluido el Japón) contengan más de las tres cuartas partes de la población mundial, si se supone un total de 6130 millones de habitantes.9 De hecho se estima que más del $85 \%$ del incre. mento de la población mundial entre 1965 y el año 2000 tendrá lugar en las áreas menos desarrolladas, ${ }^{10}$ debido a la expectativa de altas

5 Para estas cuatro regiones en su conjunto el incremento fue de $116 \%$; según la definición más rigurosa (véase la nota 3 ), fue đe $138 \%$. Ibid., cuadros 2 y 11 .

6 Ibid., cifra derivada del cuadro 1.

7 Ibid., cuadros 7-9. La definición más rigurosa de "áreas menos desarrolladas" reduce la proporción de éstas en la población total de las ciudades de más de 500000 habitantes a $39.5 \%$, y la proporción de habitantes de estas ciudades al total de la población urbana a $43 \%$ (cuadro 11 ).

8 Ibid., cuadro 9. Alrededor del $25 \%$ estaba en ciudades de más de 100000 habitantes.

9 Proyección intermedia. Véase John D. Durand, "The Modern Expansion of World Population", Proceedings de la Sociedad Filosófica Norteamericana, Vol. CXI, junio de 1967, cuadro 1.

Io Ibid., cuadro 5. 
tasas de natalidad en esas áreas, reforzada por la mortalidad en descenso -en contraste con las tendencias de crecimiento demográfico más lento que prevalecen en los países industriales. Con base en las tendencias y la experiencia recientes, y en los muchos factores que atraen a la gente a las ciudades y la inducen a emigrar de las áreas rurales a las pequeñas poblaciones, es posible que para el año 2000 cerca de la cuarta parte de la población mundial viva en ciudades de 500000 o más habitantes; de estos 1500 millones de personas, tal vez tanto como las dos terceras partes, o casi 1000 millones, se encontrará en las regiones menos desarrolladas. Y de estos mil millones de personas, es fácil que 300 millones estén localizados en América Latina. Si la línea divisoria se sitúa en 100000 , es probable que las áreas menos desarrolladas contengan más de 1800 millones de habitantes en ciudades de ese tamaño y más, de los cuales unos 400 millones se encontrarán en América Latina.

América Latina parece estar tomando la delantera, desde el punto de vista de las tasas de incremento, pues es posible que casi el $47 \%$ de su población habite, para el año 2000 , ciudades de 500000 o más habitantes, lo cual sería casi el doble de la proporción en el mundo como un todo. $\mathrm{Y}$ es probable que aproximadamente el $60 \%$ de la población futura de América Latina habite ciudades de más de 100000 habitantes cada una. Ya en 1960, 19 ciudades de América Latina contenían más de medio millón de personas; hoy en día hay por lo menos 22 ciudades semejantes, y habrá muchas más en el futuro según continúe la migración rural-urbana y la gente fluya de las localidades más pequeñas a las de tamaño mediano y después a las más grandes:

La urbanización rápida es evidente a través de América Latina. Si se emplea la definición habitual en las comparaciones internacionales, de 20000 o más habitantes como localidad urbana, la población urbana de América Latina se expandió a una tasa media anual de $5.1 \%$ entre los años 1940 y 1960. Esta tasa fue de por lo menos $5.3 \%$ en 1950-1960. En este decenio, en los dos países más populosos, Brasil y México, la tasa fue de 6.5 y $5.2 \%$, respectivamente. En Venezuela, fue de $8.2 \%$; en la República Dominicana, un país pequeño, fue de $9.0 \%$; en Panamá, de 5.1 En México, la tasa de crecimiento de la población urbana en ciudades de 100000 o más habitantes, en el período 1950-1960, fue de 5.3\%; estas ciudades registraron las dos terceras partes del total de la población urbana en 1960. Las tasas correspondientes de Venezuela y Brasil fueron de 8.1 y $5.5 \% .11$ En Venezuela, el número de personas en ciudades de más de 100000 habitantes constituyó apenas el $10 \%$ de la población total en 1940, pero esta proporción ascendió hasta el $30 \%$ en 1961.12

Algunas de las ciudades más grandes de América Latina aumentaron su población en los años cincuenta en un 60 a un $70 \%$; por ejemplo, la ciudad de México, São Paulo, Bogotá, Guayaquil, Quito;

11 Datos para Brasil de John Durand y César Peláez, "Patterns of Urbanization in Latin America", Milbank Memorial Fund Quarterly, Vol. XLIII (Parte 2, octubre de 1965), cuadros 4 y 5 . Datos para México, Venezuela, Panamá y República Dominicana de Carmen Miró, "The Population of Latin America", Demo graphy, Vol. I, Núm. 1, 1964, cuadro 8.

12 Lander y Funes, loc. cit., cuadro 13. 
y otras casi la duplicaron o más, como Caracas, Lima, Cali, Santo Domingo, Monterrey, Belo Horizonte, Guadalajara. En ciertas ciudades más pequeñas, se registraron tasas de crecimiento más elevadas. En Buenos Aires, la capital más grande de América Latina, y en Río de Janeiro, la antigua capital de Brasil, la expansión fue menos rápida, pero en estas dos ciudades el tamaño por sí mismo ya es significativo, de manera que aun un crecimiento anual de $3 \%$ es importante $y$ debe tomarse en cuenta.

Es probable que continúen, por lo general, las tendencias actuales en América Latina, aunque las tasas de urbanización puedan disminuir en algunos de los países más grandes así como en las capitales mayores. No es probable que se aminore la migración rural-urbana, pero puede dirigirse, en forma creciente, a las ciudades de tamaño medio según se extienda a ellas el desarrollo industrial y comercial. La perspectiva mínima que se tiene es que la población urbana en su conjunto se duplicará cada quince años, mientras que la población rural, en el mismo período de tiempo, puede que ascienda en sólo un $40 \%$ (cifra que por sí sola es inquietante). En países como Ecuador, Colombia y algunas naciones de Américā Central, la expansión será de tres tantos cada quince años, y en otros, como la República Dominicana y Venezuela, de cuatro o cinco veces, si persisten las tendencias presentes..$^{13}$

Los datos sobre la población mundial, sobre todo de las áreas menos desarrolladas, están sujetos a revisión y deben, desde luego, tomarse como estimaciones. Las aproximaciones son aún más grandes en el caso de la población urbana, para la cual hay diferentes definiciones, y un poblado de un tamaño dado en función del número de habitantes tiene obviamente un significado bastante diferente en Africa que en América Latina. Además, las proyecciones al año 2000 no. son mejores que las premisas en las que estén basadas. No obstante, parece necesario tener alguna idea de las magnitudes, no sólo importantes en sí mismas sino también como trasfondo para otras consideraciones que afectan el crecimiento urbano y le dan características peculiares en nuestro tiempo.

\section{III}

Las ciudades de América Latina que crecen con rapidez - y lo mismo es verdad, en términos generales, de las otras áreas menos desarrolladas - no son el producto de sociedades agrícolas e industriales altamente productivas, sino que se relacionan con condiciones en donde la productividad rural es, por lo común, baja; la industria fabril está desarrollada sólo en parte; los niveles de educación, calificación, salud y seguro social son todavía sumamente inadecuados, y el ingreso y la propiedad están altamente concentrados. Las ciudades de América Latina - aun las más grandes de origen europeoson pobres; pobres $\mathrm{y}$ en parte desatendidas o pobres $\mathrm{y}$ atrasadas, o pobres en promedio pero con manchas de riqueza entremezcladas en barriadas pasmosamente horrendas y alrededores repulsivos, más o menos unidas por transportación y otros servicios crecientemente

13 R. Utria, loc. cit., p. 266. 
inadecuados. Tlas naciones de América Latina están siendo acosadas por la "ciudad prematura" -una visión previa de la futura "no ciudad" si es que no se cambian las perspectivas presentes de desarrollo.

Es del conocimiento general que en los alrededores de casi todaś las ciudades grandes de América Latina han surgido las comunidades llamadas "marginales", que a veces han penetrado hasta su centro. Éstas varían desde los tugurios hasta las poblaciones "callampas" y las subdivisiones clandestinas, y constituyen lo que un autor llama asentamientos urbanos no regulados, en su mayor parte inevitables.14 Estos asentamientos son o han sido los "centros de recepción", en términos generales; de los migrantes pobres y no calificados procedentes de las áreas rurales y de las localidades urbanas menores. La expansión de estas comunidades ha sido tan rápida y tan inesperada que ha sido imposible proveerlas de los servicios esenciales -agua, drenaje, luz, y los otros servicios municipales habituales; carecen de escuelas, unidades de salud, protección y amenidades; los terrenos en que están establecidas están sujetos, con frecuencia, a la erosión y a las inundaciones; sus viviendas consisten, en lo principal, en chozas forjadas, de manera ingeniosa, con láminas metálicas de desecho, madera, piedra, o tabla; a veces tienen una organización "cívica" propia, pero también con demasiada frecuencia abrigan a los criminales habituales y procrean la violencia, el robo y el vicio. A estas áreas se deben añadir los tradicionales tugurios empotrados en el centro de las ciudades, tal vez hoy día en peores condiciones que antes. Los moradores de las barriadas y de las comunidades marginales quizás abarquen de la cuarta parte a la mitad o más de la población de las ciudades más grandes de la India, Turquía, Perú, Venezuela, Irak, Senegal y muchos otros países.

Estas subciudades de poblaciones marginales son el resultado de dos dificultades principales: por un lado, la incapacidad de las áreas rurales para proveer medios de vida a una población que crece con rapidez; por el otro, la inhabilidad del sistema económico para absorber a los habitantes urbanos, de manera suficiente, dentro del empleo industrial. ${ }^{15}$ Ambos problemas requieren cierta elaboración y a través de ellos corre un tercer factor, a saber, los niveles relativamente altos de fecundidad combinados con una mortalidad. general e infantil, en descenso, lo que por el momento significa tasas de crecimiento de la población sin precedente que probablemente perdurarán por largo período de tiempo. ${ }^{16}$

14 J. F. C. Turner, Uncontrolled Urban Settlements, trabajo presentado al Seminario Interregional citado en la nota 1 . Este escrito contiene un análisis excelente de los problemas que hay que tratar, con ejemplos de muchas partes del mundo menos desarrollado. Véase también $\mathrm{R}$. Utria, loc. cit.

15 R. Utria, loc. cit., passim, y División de Población de las Naciones Unidas con la colaboración del Prof. Sidney Goldstein, Urbanization and Economic and Social Change, Seminario Interregional citado en la nota 1.

16 Una encuesta que se llevó a cabo en siete capitales de América Latina demostró un número promedio de hijos nacidos vivos por mujer de edad fecunda que iba de 2.25 en Río de Janeiro a 3.27 en la ciudad de México (dejando fuera a Buenos Aires, donde fue 1.49). Véase Carmen Miró, "Some Misconceptions Disproved: A Program of Comparative Fertility Surveys in Latin America", en B. Berelson, compilador, Family Planning and Population Programs (Chicago, 1966), p. 639, cuadro 2. 
Las condiciones rurales son responsables, en la mayor parte de los países, de una porción considerable de la migración a los asentamientos no regulados en las ciudades más grandes. A pesar del progreso logrado en muchas áreas, los sistemas de tenencia de la tierra son inadecuados $\mathrm{y}$, con frecuencia, injustos desde el punto de vista social. Los más de los campesinos no poseen tierra, o tienen parcelas tan pequeñas, en propiedad o en arriendo, que no pueden ganarse la vida. Los programas para mejorar los métodos de cultivo y elevar los rendimientos, ampliar los mercados y proveer incentivos no han sido suficientes. La gente se mueve hacia los centros urbanos, no porque las técnicas nuevas la hayan hecho superflua en las explotaciones agrícolas, como en los países avanzados, sino porque la tierra no puede alimentarla. La gente va en busca de trabajos mejor pagados, de nuevas oportunidades o de la seguridad aparente - $\mathrm{o}$ aun el "atractivo"- de la ciudad. Sería imposible analizar aquí en detalle estos problemas. Existe conciencia creciente de ellos, y muchos programas de desarrollo agrario y de cultivos se están llevando a cabo en Amé rica Latina, India, Pakistán y otros lados. Pero es dudoso que el alcance de estos programas sea suficientemente amplio. ${ }^{\top} \mathrm{Y}$ debe admitirse que, según tengan éxito, la conclusión lógica, al aumentar la productividad y los ingresos, sería que más gente se desplazara hacia ocupaciones no agrícolas. En esto, ayudaría el que se establecieran nuevas industrias fabriles en las áreas donde hay exceso de población agrícola, alrededor de las poblaciones más pequeñas.

El desarrollo industrial, que por lo general y de manera necesaria está restringido a las ciudades, todavía no ha alcanzado una base lo bastante amplia para afrontar las adiciones potenciales a la fuerza de trabajo industrial que están resultando de la migración a las ciudades, o aun para absorber el crecimiento natural de la población urbana en edad de trabajar. En 1960 se encontró que en América Latina la proporción del empleo fabril, en relación con el total del empleo no agrícola, fue de $27 \%$ contra $35 \%$ veinte años antes, y que el empleo en las actividades terciarias es relativamente más alto en la región que en los Estados Unidos y Europa en una etapa semejante de industrialización. ${ }^{77}$ Esto significa que en América Latina el empleo ha aumentado con más rapidez en los servicios, el comercio y en muchas otras ocupaciones menores que en la industria, lo que sugiere que el fluio de migrantes a las ciudades ha resultado en una propagación del subempleo urbano. Una estimación señala que en América Latina, en 1960, 8.2 millones de personas - el $12 \%$ de la fuerza de trabajoestaban en condiciones de "desempleo disfrazado", y que en vez de disminuir, se puede esperar que este sector de la vida urbana -improductivo y con ingresos de subsistencia - llegue a constituirse en cerca de 11 millones de personas para 1970.18 En algunos países, se estima que el subempleo alcance a ser el $25 \%$ del empleo total en

17 Comisión Económica para América Latina, El proceso de industrialización en América Latina, Anexo estadístico, Santiago, Chile, 1966, pp. 11 y 13.

$18 \mathrm{~B}$. Hopenhayn, Ocupación y desarrollo económico en Ámérica Latina, ILPES, Santiago, 1966, citado por F. H. Cardoso y J. L. Reyna, "Industrialization, Occupational Structure and Social Stratification in Latin America", en Cole Blasier, compilador, Constructive Change in Latin America, University of Pittsburgh Press. 1968, nota 11, p. 54 . 
"servicios misceláneos", lo cual comprende una parte importante de la actividad terciaria.19

A pesar de sus tasas altas y de los avances espectaculares en ciertos ramos, el desarrollo industrial en los países de América Latina no es todavía lo suficientemente rápido o diversificado, ni se están expandiendo a su vez de manera adecuada los servicios que le dan apoyo - -educativos y de adiestramiento- como para absorber en empleos productivos y constantes las adiciones a la población urbana en edad de trabajar. En particular, hay grandes excedentes de trabajo no calificado, incluida mano de obra femenina potencialmente empleable. El desarrollo de la tecnología moderna tiende, además, en muchos casos, a aumentar la relación capital/trabajo y, de cualquier manera, a requerir personal altamente calificado. LAquí, de nueva cuenta, no sería posible intentar un análisis más a fondo del cuadro del desarrollo industrial, excepto mencionar que además de los limitados mercados internos --mercados que en su mayor parte son el resultado de la baja productividad agraria- existen otros numerosos problemas relacionados con las condiciones industriales y de comercio, acompañadas, por lo general, de los de estructura y fluctuación del comercio internacional, que afectan de manera desfavorable los programas y las políticas de desarrollo de las naciones más pobres.

LEn el fondo de la expansión de las comunidades marginales y las poblaciones "callampas" de las ciudades de América Latina está entonces, por un lado, el desequilibrio entre la productividad industrial y la agrícola, y por el otro, la dificultad de perseguir, a un mismo tiempo, el desarrollo adecuado de la agricultura y el crecimiento más acelerado de la industria. Estas comunidades viven y crecen con velocidad. Tales "grupos marginales", según una estimación, crecen en ciertos lugares a tasas hasta de $15 \%$ anual. ${ }^{20}$ Esto produce presiones no sólo sobre el empleo, sino también sobre los salarios, y tiende a abaratar el trabajo por debajo de los niveles de subsistencia. Se establece, además, una carga imposible sobre los servicios urbanos, y se abre una fuente creciente de inestabilidad política. Así, las condiciones de vida en las ciudades reflejan -y por largo tiempo continuarán haciéndolo- las condiciones económicas angustiosas no sólo de sus habitantes sino de las economías subdesarrolladas en su conjunto.

El crecimiento adecuado de la ciudad, de la vivienda y de los servicios sólo puede ser el fruto de una elevada productividad y de una producción en ascenso acelerado, combinados con un sistema educativo eficiente, una estructura impositiva equitativa, una mejor distribución de los ingresos, una estructura social menos rígida, y uná conciencia cuidadosa del conjunto de factores que afectan a la ciudad. La expansión de la capa de ingresos medios en los países en vías de desarrollo, aunque genera demanda de gran parte de lo que una ciudad moderna debe ser, compensa muy poco el peso creciente de la oferta de entrantes a las comunidades marginales. Así, por cada edificio de clase media o rascacielos para oficinas que se construya, pueden surgir de un día para otro miles de chozas habitadas por cinco, seis o

19 Ibid., p. 44.

20 Felipe Herrera, presidente del Banco Interamericano de Desarrollo, en un discurso pronunciado en la Universidad de Salvador, Bahía, Brasil, 23 de septiembre de 1967. 
más personas en cada cuarto. Estas personas son analfabetas, hambrientas, enfermizas y necesitadas, con pocas oportunidades para avanzar económica y socialmente. Según un estudio, el $90 \%$ de los migrantes a Santiago de Chile no logra desarrollar movilidad ascendente.21 Se pueden dar incontables ejemplos acerca del desempleo, los bajos ingresos y las condiciones miserables de vida en los asentamientos urbanos no regulados de América Latina y otros lados.22

\section{IV}

Además de la extensión creciente de la ciudad subdesarrollada, los sistemas grotescos de transportación, el ruido y la actual propagación rápida de la contaminación atmosférica, la escasez de agua, las inundaciones, la ausencia casi total de servicios de policía, la suciedad, y lảs disformes y no planeadas aglomeraciones de construcciones, destaca un problema principal: la vivienda. Se ha sostenido que el problema de la vivienda urbana en los países menos desarrollados es insoluble. Esta es una afirmación intuitiva y a priori que muchos sin duda discutirían. No obstante, puede que no sea demasiado desatinada. Algunas estimaciones recientes para América Latina - y con seguridad se han hecho cálculos semejantes sobre otras áreas-señalan que el déficit de aproximadamente 7 millones de unidades de vivienda urbana, aun en función de las presentes definiciones inadecuadas, podría ser reducido de manera relativa -es decir, en relación con el conjunto de necesidades habitacionales- dentro de este siglo, pero de ninguna manera eliminado. ${ }^{23}$

Las tasas actuales de construcción de viviendas son, en su mayor parte, bajas. En 1964, una estimación señaló la construcción en América Latina de poco más de 400000 unidades, urbanas y rurales, que representa 2 unidades nuevas por cada 1000 habitantes. Otra estimación apunta que la proporción pudo haber sido de 3.24 En particular, México, Venezuela y Brasil se están quedando atrás en la tarea de hacer frente a las necesidades anuales de vivienda. Chile, Costa Rica y Colombia están cercanos a encarar el incremento anual de la demanda. A pesar de los nuevos programas y de los nuevos medios de financiamiento interno y externo, es poco probable que se progrese mucho más allá de las tasas de 1964. Por consiguiente, es dudoso que se esté reduciendo el déficit. Tan sólo para evitar que el déficit aumente, suponiendo un espacio medio mínimo por unidad habitacio-

21 Centro Latinoamericano de Demografía, Encuesta sobre inmigración en el Gran Santiago, citado por Teresa Orrego Lyon, "Algunas consideraciones sobre marginalidad urbana", Temas del BID, Banco Interamericano de Desarrollo, Washington, Núm. IV, septiembre de 1967, p. 30.

22 R. Utria, loc. cit., passim, cita varios ejemplos: véanse también los trabajos presentados al Seminario Interregional de Pittsburgh, citado en la nota 1. Se puede encontrar un estudio comparado reciente e interesante sobre las barriadas de América Latina en Lloyd H. Rogler, "Slum Neighborhoods in Latin America", Journat of Inter-American Studies, Vol. IX, octubre de 1967, pp. 507-528.

23 R. Utria, loc. cit., pp. 260-263.

24 Ambas estimaciones son citadas en una reseña bien meditada de la cuestión publicada recientemente por el Banco Francés e Italiano para América del Sur, "El problema de la vivienda en América Latina", Estudios Económicos, I (1967), pp. 49-67. La proporción por mil habitantes en Europa Occidental fue 7.4 en 1961. 
nal, América Latina tendría que construir anualmente varias veces más unidades que las que construye ahora; tal vez seis veces más que la tasa actual de construcción, para llegar a una proporción de diez unidades por mil habitantes. Para lograr esto, el costo de la inversión anual, estimado conservadoramente (incluidos servicios e instalaciones básicos), alcanzaría a casi el $40 \%$ de la actual inversión bruta global (que equivale a más o menos el $16 \%$ del producto bruto).$^{2 \bar{s}} \mathrm{Si}$ se dan ciertas condiciones, esto tal vez no sería imposible, aunque sí extremadamente difícil. Todavía significaría conllevar en forma indefinida el déficit urbano de 7 millones, así como el déficit rural (que se ha estimado entre 8 y 14 millones de unidades). Para reducir realmente el déficit, al menos en las ciudades, se requeriría sin duda un esfuerzo mucho mayor y una proporción más grande de la inversión bruta anual. ${ }^{26}$ Se podría especular acerca de las diversas posibilidades de financiamiento $\mathrm{y}$ hacer suposiciones acerca de las tasas de crecimiento, los coeficientes de ahorro, las cargas impositivas, las especificaciones de las viviendas, etc. Pero sólo es necesario recordar que además de la vivienda urbana (y rural), existen otras tareas que hay que desempeñar -en la educación, la tenencia de la tierra, el desarrollo agrícola, el saneamiento y el bienestar, y otras urgentes necesidades sociales y económicas, en América Latina y en otros lados.!

El problema, evidentemente, no consiste sólo en obtener recursós financieros o aun reales, ni mucho menos se trata de uno que pueda resolverse con la asistencia de la cooperación financiera internacional. El problema es, también, de conceptos básicos. "Se observa más bien una actitud contemplativa frente al progresivo crecimiento del déficit de servicios habitacionales y comunales y aparente intención urbanístico-ornamental en el tratamiento de la planificación urbana".27 Pero la planificación urbana en sí misma, en el sentido de planes operativos y no de sueños de los arquitectos, casi no existe. Donde desde la nada se ha planeado y se ha desarrollado una ciudad como en el caso de Brasilia, se ha resuelto poco o ninguna cosa. El desarrollo de la vivienda sufre, en consecuencia, de la insuficiencia de un planeamiento global. Predominan las soluciones ad hoc, y aun los mejores programas de vivienda atraviesan dificultades y demuestran ser insuficientes en relación con la escala de los problemas de la ciudad.

También por estas razones, el diseño de los programas urbanos y de vivienda es poco realista en función de las fuerzas que influyen el desarrollo urbano. La mayor parte de los programas públicos y privados de vivienda en los países menos desarrollados en efecto están tratando de hacer frente, en el mejor de los casos, a los requisitos de ciertos tipos de demanda de clase media, y aun en las capas medias bajas las normas y los materiales empleados tienden a ser aquellos que corresponden a los países más ricos. (Los costos de la vivienda son altos en relación con el nivel de desarrollo y el poder adquisitivo. Una mera comparación de los costos de la mano de obra y los materiales entre, digamos, California y Asunción, Paraguay, simplemente no cabe, porque de hecho en este último país tales costos, aunque más bajos,

25 R. Utria, loc. cit., p. 263.

26 La fuente que se citó en la nota 24 contiene cálculos alternativos por varios autores. El problema queda igualmente insoluble.

27 R. Utria, loc. cit., p. 272. 
pueden ser más elevados en. función de los ingresos del asalariado y del ingreso real previsible. Los intereses sobre los préstamos de vivienda son, por lo general, más altos en los países menos desarrollados, tanto en términos absolutos como relativos.

Se relaciona de manera muy estrecha con ello la cuestión del valor de los terrenos. Las estimaciones del costo de los programas de vivienda en América Latina, tan sólo para contener el problema, toman poco en cuenta el costo real de los terrenos. En el caso de la unidad mínima de vivienda, en un edificio multifamiliar de cuatro pisos, con un promedio de 80 metros cuadrados de terreno por unidad de 70 metros cuadrados de piso, el costo del terreno y los servicios básicos sería de por lo menos el $9 \%$ del costo total de la unidad; para una familia de ingresos medios, el costo iría del $10 \%$ por 80 metros cuadrados de piso a un $20 \%$ por el doble de esa cantidad de construcción por unidad. ${ }^{2 s}$ Como resultado de la especulación y de las preferencias de inversión, la falta de reglamentación y factores económicos generales, los valores de los terrenos en las ciudades de América Latina son notoriamente altos. En Caracas, los valores de los sitios en diferentes partes de la ciudad aumentaron de 4 a 18 veces en un período de trece años. 29 "Hoy en día no hay oferta de terrenos en Caracas que estén al alcance de las familias de ingreso medio y bajo, y aun los precios de los terrenos no urbanizados son tan altos que se han vuelto prohibitivos para ser utilizados en la vivienda de bajo costo". ${ }^{30}$ En las ciudades de América Latina es común que las familias de bajo y medio ingreso tengan que pagar más por el sitio que por la unidad habitacional que se construya en ella. En la mayor parte de los países, según el Banco Interamericano de Desarrollo, hay considerables dificultades legales y de otro tipo en la adquisición de terrenos para los proyectos de vivienda, además del costo.

Los programas de vivienda pública deberían comprender arreglos institucionales adecuados, desde una ley básica, servicios de investigación, y la coordinación del trabajo de los diversos organismos dentro de un plan global, hasta las relaciones convenientes con otros aspectos del desarrollo urbano y regional. En la mayor parte de los países de América Latina, estos programas son gravemente deficientes. La situación deja que desear tan sólo por lo que hace a eficiencia en la construcción y la investigación sobre nuevos materiales y métodos para reducir los costos. Con excepción de siete u ocho países que están llevando a cabo tales investigaciones, el resto de los países de América Latina continúa construyendo con métodos anticuados. ${ }^{31}$

Dada la distribución de los ingresos que prevalece en la mayoría de los países menos desarrollados, una importante proporción de los ocupantes potenciales no podría pagar, de todas maneras, dentro de un período razonable, tanto la casa como el terreno, o aun la cons-

28 Ibid., pp. 250-251, cuadro 1. Debe subrayarse que estos datos son usados como promedios en una solución "planeada" del déficit de vivienda. Por lo general, el valor de los terrenos es más alto en proporción al costo total, sobre todo en relación con la vivienda para una familia de clase media, donde puede llegar a ser hasta del $50 \%$ o más.

29 Lander y Funes, loc. cit.

30 Ibid., p. 98.

31 De un informe del Banco Interamericano de Desarrollo. 
trucción por sí sola. No está claro hasta qué grado los esfuerzos para reducir el costo por unidad habitacional podrían hacer frente a los niveles bajos de demanda a los precios de mercado y bajo condiciones financieras ordinarias. Con frecuencia los proyectos de vivienda de bajo costo sufren de cambios rápidos en la ocupación debidos a la falta de pago, y los propietarios o los inquilinos tienden a sobrepoblar sus habitaciones $y$, en parte, a usarlas para fines comerciales.

For consiguiente, una consideración adecuada del problema de la vivienda no puede abstraerse de las condiciones económicas generales ni del marco tecnológico, cultural y social. El abordar el problema en forma meramente cuantitativa es insuficiente, por más que aparenten ser asequibles las metas cuantitativas. El problema de la vivienda parece suscitar graves dudas acerca de la vida urbana en general, pero esto último es a su vez, en gran parte, reflejo de un desarrollo económico inadecuado vinculado a un crecimiento demográfico excesivamente alto. La respuesta al desarrollo urbano, y el futuro de la hoy ciudad subdesarrollada, debe buscarse, en consecuencia, dentro del contexto más amplio del crecimiento económico y del cambio social, antes que en la ciudad misma o en su estructura.」

\section{V}

En los países industrialmente avanzados se despliegan esfuerzos bastante definidos y concentrados para aplicar el conocimiento y la habilidad organizativa al aumento de la producción total y a la extensión de los beneficios de la productividad al consumo popular; esto es verdad en diferentes sistemas sociales, incluido el soviético. En su conjunto, las naciones menos desarrolladas no parecen estar alcanzando a los países industriales. Están siendo inducidas por las comunicaciones modernas - la publicidad, la radio y la televisión, el cine y las publicaciones periódicas - a adoptar las aspiraciones y los patrones de consumo de los países de altos ingresos. También, de manera parcial, incorporan la tecnología nueva a muchas actividades, con demasiada frecuencia sin tomar en cuenta las condiciones locales del mercado de trabajo, condiciones que favorecen el establecimiento de plantas industriales que trabajen a base de uso extensivo de la mano de obra en vez de alta densidad de capital. Dentro de sus mercados internos discontinuos y fragmentados, a menudo pequeños, permiten que libremente se tomen decisiones irracionales sobre las inversiones privadas que conducen a una producción industrial de altos costos y de baja absorción de fuerza de trabajo. Los programas educativos $\mathrm{y}$ otros sociales son inadecuados y son frenados por el progreso económico mismo. Las condiciones del mercado mundial no favorecen a los países menos desarrollados, y acerca de las actitudes internacionales ante el desarrollo, a pesar de lo mucho que se ha hablado - por ejemplo, en las Naciones Unidas, las conferencias regionales cumbres y otras reuniones - sólo se puede decir que dejan mucho que desear, en el mejor de los casos. La situación política internacional no es menos decepcionante, en tanto las potencias de la edad nuclear y del espacio vayan implicando a las naciones más pobres en sus rivalidades. El desarrollo bajo estas condiciones será ciertamente milagroso. 
Y las ciudades sólo pueden ser una parte de ese conjunto, y no una entidad aparte. Él desarrollo urbano sólo podría empezar a ser racional en respuesta a un mejoramiento de las condiciones generales que favorezcan el crecimiento y el cambio social.

Se ha dicho que la mayoría de las llamadas soluciones a los problemas del desarrollo urbano no son más que respuestas parciales a corto plazo a cuestiones mal planteadas. Se necesita de mucha investigación para ayudar a obtener mejores evaluaciones en las que se puedan basar las políticas. Pero, así como en casi todos los otros aspectos del desarrollo, las políticas tienen que establecerse aun en la ausencia de una información y un análisis completos y adecuados. Sobre todo, como muchos han señalado, se necesita adoptar una "estrategia". No puede haber respuestas parciales, ni se pueden trasplantar totalmente los proyectos exitosos de un país a otro. A menudo es el planificador - usando este término en su sentido amplio- quien hace que sea imposible el logro de un plan, porque o apunta demasiado alto o trata de alcanzar demasiados objetivos al mismo tiempo. Este es particularmente el caso del planeamiento urbano, donde las consideraciones sociales o aun estéticas pueden oscurecer la realidad económica. Pero también es la falta de una visión global la que en repetidas ocasiones impide que los programas individuales tengan éxito o que muestren el camino hacia soluciones más amplias.

En los países subdesarrollados parece necesario reconocer que no es probable que ciertos factores condicionantes cambien durante mucho tiempo. Cualquiera de las políticas que se siga debe entonces tomar muy en cuenta estos factores. El crecimiento demográfico, aun con programas de planificación familiar intensificados, está destinado a continuar a una elevada tasa, y se puede esperar que la migración rural-urbana presione de manera creciente sobre los servicios urbanos. $\mathrm{L}$ La expansión de las ciudades es sin duda una condición necesaria de la industrialización, y el crecimiento industrial es el instrumento principal con el cual se puede acelerar el desarrollo y aumentar la movilidad social. Pero dada la naturaleza y el nivel de los recursos humanos y físicos de un país menos desarrollado y las oportunidades educativas efectivas que probablemente se van a presentar, se debe determinar una política amplia que permita impedir el tipo de concentración que ahora está ocurriendo en las ciudades más grandes. Los países más pobres no están preparados en el orden económico e institucional para tal concentración. Una parte esencial de la estrategia debe ser, por lo tanto, fomentar el crecimiento de las ciudades más pequeñas a través del desplazamiento de industrias fabriles y otras actividades modernas por medio de incentivos apropiados y de una planeación regional conveniente. Hasta hace poco, las comunicaciones inadecuadas eran una fuerza potente que tendía a concentrar a la industria en las ciudades capitales o en otras poblaciones grandes. Pero los nuevos métodos de transportación están aportando nuevos módulos de ubicación. Se necesita que este proceso sea estimulado, como medio de aminorar o de extender hacia afuera la migración rural-urbana, y también para elevar los ingresos en los centros urbanos más pequeños hasta un nivel en que la vivienda y otras mejoras puedan estar dentro del alcance parcial del mercado 
local. Tal política no frenará la urbanización tomada en su conjunto, pero por lo menos evitará una alta concentración.

Mientras tanto, dados ciertos supuestos, tal vez excesivamente escépticos, acerca de las tendencias actuales del progreso social en los países menos desarrollados, algunos aspectos de la realidad urbana se deben encarar de manera directa. Los grupos marginales de bajo ingreso persistirán por largo tiempo; ellos crecerán por la migración rural-urbana y por las altas tasas de natalidad; no se pueden considerar como provisionales o como si estuvieran a punto de convertirse en estratos convencionales de clase media. Los planes de desarrollo urbano, por lo tanto, deben incluir la integración y mejoramiento gradual y selectivo de estos grupos. Se empieza a reconocer esta parte de la estrategia en muchos lugares y en algunos países se ha vuelto explícita. Existen diversos medios para darle contenido. El arrasar los tugurios, como sucede en los países industriales avanzados, sólo es aplicable en las áreas más pobres del centro de las ciudades grandes, pero no en los llamados asentamientos urbanos no regulados o en las barriadas marginales. La existencia de las últimas y las condiciones que las crean no pueden ser ignoradas. Por lo tanto, parece necesario adaptar ciertas instituciones a su existencia e introducir en esos asentamientos elementos importantes de mejoramiento en los cuales los colonos puedan participar de manera activa.

En muchos casos, sería recomendable legalizar los "derechos de las poblaciones callampas", sobre todo donde la colonia es más que un lugar provisional de morada y demuestra síntomas claros de "mar- cha progresiva", según las características ocupacionales de sus habitantes, sus actitudes ante la posesión legítima y su participación en la actividad comunitaria. El establecimiento de la posesión legítima debería ir acompañado de la introducción subsidiada de servicios municipales básicos, con frecuencia con la participación local en su construcción a través de trabajo voluntario. Son requisitos esenciales para tales programas los programas cooperativos subsidiados para la venta de materiales, la asistencia técnica gratuita sobre la construcción, y la ayuda en la mejoría de las condiciones de la vida familiar y del hogar. Ha habido muchos experimentos de este tipo en América Latina, Africa y Asia, incluyendo algunas en que se proveen "casasnúcleo" para que se amplíen de manera gradual. El proyecto de "préstamo de techo" en Ghana, los planes de autoayuda en Santiago y Bogotá, y muchos otros de naturaleza semejante parecen marchar en la dirección correcta. Todos suponen que el colono sea capaz de una actividad organizativa y de una conducta en gran medida responsable. "El asentamiento irregular no debe entenderse como un fenómeno totalmente lamentable... En algunos aspectos, es probablemente la forma más significativa de construir hogares que está aconteciendo en el mundo de hoy." 32 Así, parece haber un volumen grande de ahorro no aprovechado, en el sentido de esfuerzo potencial, entre los colonos de esos asentamientos, de tal manera que los recursos públicos se pongan a trabajar de manera conveniente en esa área. ${ }^{33}$

32 De un "Profile" de Charles Abrams, en The New Yorker, 1967.

33 Los mejoramientos en los asentamientos irregulares podrían también tomar la forma de ciertos servicios "colectivos" para compensar la necesidad de la vi- 
Con frecuencia la legitimación de los derechos de los colonos usurpadores puede requerir la compra franca del terreno por una autoridad local, a través de un programa financiado nacionalmente, para concederlo en propiedad al colono. En algunos casos, una venta subsidiada sería preferible, o una combinación de tenencia y venta por un período de tiempo dado. Cada proyecto tendría que adaptarse a las características particulares del asentamiento. En donde los moradores comunitarios marginales son en realidad propietarios, o han empezado a pagar el terreno que ocupan y han comenzado a construir sobre él, acontece una situación un poco diferente. En estos casos, sería preciso introducir los servicios básicos y ayudar en la racionalización del proceso de la construcción y del desarrollo, sobre todo sustituir el financiamiento oneroso e inadecuado por un sistema subsidiado, adaptado a los niveles de ingreso de los moradores actuales y en perspectiva.

Una estrategia debe también incluir la consideración del problema general del valor de los terrenos, cuya incidencia, aun en los grupos de ingresos medios, es indebidamente desfavorable. La mudanza desde una vivienda derruida o de una choza a un vecindario mejor o a una colonia para personas de ingresos moderados, puede demorarse con frecuencia o aplazarse por tiempo indefinido por la influencia del valor de la ubicación sobre las rentas o sobre el precio de compra de la nueva habitación. Dadas las tasas altas de interés que prevalecen en los préstamos ordinarios para la vivienda y los períodos relativamente cortos de amortización, el propietario potencial de casa en un país subdesarrollado está en situación sumamente desventajosa. Parece haber necesidad de reglamentar con rigor los terrenos urbanos y la tenencia de la propiedad urbana no fincada, si es que los valores especulativos han de dejar de actuar como factores negativos en el desarrollo de la vivienda. El alza del valor de los terrenos en América Latina, por ejemplo, ha eliminado sin duda a millones de personas del mercado de la vivienda y ha reducido el alcance de innumerables proyectos de vivienda. Ha contribuido también a la extensión de los asentamientos no regulados. Parece ser fundamental que debe efectuarse algún tipo de "reforma urbana", en muchos aspectos paralelo a la reforma agraria. También aparenta ser inevitable, en los años que vienen, la aplicación de un fuerte impuesto sobre las ganancias de capital obtenidas de las propiedades urbanas que estén en exceso de un valor mínimo y la reglamentación necesaria para prevenir la acumulación de la riqueza urbana en manos privadas, y especialmente, la concentración de la propiedad privada en zonas urbanas habitadas por familias de bajos ingresos 1 Para que sea efectiva, la reforma urbana tendría también que restringir de alguna forma la libre venta privada por parte de los poseedores de vivienda subsidiada.

Un enfoque nuevo de los problemas del desarrollo urbano parecería exigir además una revisión de los impuestos sobre la propiedad urbana en general, sobre todo para introducir el principio de las tasas progresivas. Los elevados costos del desarrollo suburbano moderno

vienda parcial o provisional, o la adopción de normas estrechas de espacio mínimo. Pienso en dormitorios, cocinas comunales, baños, lavanderías, áreas para los niños, clubes de recreación, etc., que han sido probados en muchos lugares. 
para la clase media alta en las ciudades de América Latina deberían ser compensados por altos impuestos prediales y la fijación de impuestos especiales; $\mathrm{y}$, en general, las propiedades grandes del sector adinerado, que a menudo comprenden terrenos muy extensos, deberían sujetarse a valuaciones efectivas y a tasas progresivas. Los impuestos sobre la propiedad urbana en las grandes ciudades de América Latina, donde la capacidad impositiva es relativamente más alta en ciertos grupos, son en efecto bajos y aun nominalmente bajos:

La tasa efectiva del impuesto mexicano sobre la propiedad urbana es aproximadamente la mitad del uno por ciento del valor real o efectivo del mercado, comparada con la tasa nominal de $1.09 \%$ del valor catastral... La tasa nominal de impuestos de Caracas es de $6 \%$ de la renta potencial; en Montevideo la tasa es de $0.65 \%$ del valor catastral (con exclusión del impuesto adicional)... [pero] las tasas afectivas deben haber sido sólo una pequeña fracción de las tasas nominales. La tasa efectiva más elevada en México resulta más bien de una mejor administración que de tasas nominales más altas. [Sin embargo], los ingresos que provienen del impuesto sobre la propiedad representan aproximadamente [sólo] el uno por ciento del ingreso nacional que se origina en el Distrito Federal [de México], alrededor del doble de la proporción que se encuentra en Caracas, pero menos de la mitad de la proporción que se estima en las ciudades estadounidenses con poblaciones de un millón o más... Las tasas mexicanas de los impuestos sobre la propiedad... son modestas en comparación con las tasas en la mayor parte de las ciudades estadounidenses, y, de hecho, con las tasas prevalecientes en muchas jurisdicciones donde el nivel de desarrollo económico no es más alto que el del Distrito Federal de México. ${ }^{34}$

Así, hay amplio espacio para ulteriores aumentos de las tasas efectivas, y no existe ninguna razón válida para no introducir las tasas progresivas, aunque queda en pie alguna opinión fiscal experta a favor de la proporcionalidad. 35

Los impuestos y la reglamentación de los valores de las propiedades urbanas, y la política más amplia de reforma urbana, deberían considerarse dentro del contexto más general de la realidad de las comunidades marginales extensivas, para las cuales parecen ahora indicarse nuevas preferencias en el gasto. La reforma urbana, por lo general, puede desearse desde el punto de vista social, como paso hacia una sociedad más igualitaria, pero se necesita en especial como parte de un plan racional para el desarrollo urbano, antes de que las grandes ciudades subdesarrolladas sean aprisionadas por los asentamientos no regulados y el ideal del "cinturón verde" de las grandes poblaciones sea sustituido por un "cinturón de color café" o por un "cinturón de miseria", como ya empieza a manifestarse en muchas partes del mundo. Las autorizaciones para construir hogares lujosos en los suburbios de la clase media deberían, en razón a los intereses de los dueños mismos, restringirse o condicionarse con base en con-

34 Oliver Oldman et al., Financing Urban Development in Mexico City (Cambrigde, Mass., Harvard University Press, 1967), pp. 79-80. La referencia es a lugares en Africa, Brasil e India.

35 Los autores del libro citado en la nota anterior desaprueban fuertemente las tasas progresivas. Cf. Ibid., p. 81 . 
tribuciones al financiamiento de proyectos de colonias populares y otras en las zonas de menor ingreso o al mejoramiento de la vivienda deficiente. La ciudad subdesarrollada no puede costearse hogares millonarios al lado de millones de chozas.

Se necesita la innovación tecnológica para la ciudad subdesarrollada. Gran parte del progreso técnico en la planificación urbana y en la construcción se relaciona con lo que las comunidades opulentas de las naciones industriales avanzadas pueden hacer con sus propios recursos. Los conceptos de la planificación urbana, como la tecnología industrial, parecen requerir una adaptación a un marco de referencia menos desarrollado. Con frecuencia, las propuestas de los planificadores urbanos, ricas en imaginación y en ideales humanísticos, están vacías en lo que se refiere al contenido económico; se relacionan de manera insuficiente con las condiciones iniciales o con las posibilidades sociales actuales de llevarlas a cabo, y no cuantifican las muchas variables que entran en juego para obtener resultados, ni toman en cuenta las alternativas económicas más amplias. El planeamiento urbano es parte de un proceso; por sí mismo es necesariamente incompleto.LEs indispensable asociar a los planificadores de manera más estrecha con los economistas y los sociólogos preocupados del desarrollo urbano, ya que una totalidad de factores económicos y sociales deben entrar en el cuadro. En particular, la economía de la ciudad subdesarrollada, a diferencia de la ciudad opulenta de las naciones occidentales industriales, debe convertirse en el objeto de un estudio cuidadoso, y todo el sistema de interrelaciones del desarrollo urbano y del desarrollo económico y social global necesita esclarecerse.

Una mayor conciencia de estos problemas en los países menos desarrollados está destinada a llevar a adoptar políticas nacionales de desarrollo urbano en que puedan establecerse los mecanismos adecuados para someter las políticas de crecimiento y de vivienda de las principales ciudades al alcance de las autoridades centrales o federales, sin restringir de ninguna manera las funciones locales ni contener la iniciativa local. Las naciones industriales se mueven en esta dirección, y ahora mucha de la preocupación de los planificadores tiene que ver con el desarrollo urbano como parte integral del desarrollo nacional. Los países menos desarrollados, en vista de sus perspectivas demográficas y de sus tendencias en la urbanización, anteriormente esbozadas, tienen necesidad igual, si no mayor, de conformar políticas nacionales. $\lfloor$ El crecimiento urbano, la industrialización y la planeación regional no pueden considerarse hoy día como procesos independientes. J La expansión y la modernización de la transportación, dentro de las ciudades y en el plano interurbano, tiene que formar parte integral del mismo proceso y de las mismas políticas. Se necesitan urgentemente, en los países más pobres, nuevas prioridades en el gasto público que reflejen estos objetivos.

Las ciudades subdesarrolladas a menudo son proyectadas hacia el futuro no con base en lo que son y lo que probablemente serán, dadas sus condiciones fundamentales presentes, o con base en un proceso realista de cambio, sino de acuerdo con los modelos de las ciudades ya pasadas de moda de las naciones industriales desarrolladas.j Esto sucede de igual manera en estas últimas naciones, en donde los que 
hacen la política "ocasionalmente han estado tratando de resolver los problemas de la ciudad que ya pasó".36 Se debe admitir que cualquier pronóstico es atrevido, pero la mayor parte de las proyecciones tienden a ser extremadamente conservadoras. Las proyecciones que se basan en las condiciones presentes pueden ser demasiado pesimistas. "Los sociólogos y los economistas que fundamentan sus proyectos para una expansión futura económica y urbana con base en las fuerzas que actúan en el presente, proyectando sólo aquellos cambios que pueden resultar de un aceleramiento de tales fuerzas, tienden a arribar a una megalópolis universal, mecanizada, estandarizada, efectivamente deshumanizada, como meta última de la evolución urbana." 37 Este día del juicio urbano no es todavía la forma de ver las cosas de los sociólogos, los economistas o los planificadores urbanos en las áreas menos desarrolladas _-más bien demuestran una conciencia insuficiente de las implicaciones de la urbanización-, pero ciertamente es común una proyección de las condiciones presentes. Un plan director para Monterrey, México, que se publicó recientemente, donde se estima que la población va a crecer del millón actual a más de 5 millones para el año 2000, no toma en cuenta ninguna alternativa al automóvil privado o al autobús como medio de transporte interno, aunque el plan está bien concebido en casi todas sus partes. ${ }^{38}$ Pero ¿serán necesarios los automóviles, como hoy los conocemos, el año 2000? Es posible que aun un sistema de ferrocarril subterráneo sea obsoleto para entonces. No es necesario que los expertos sobre el desarrollo urbano se sumerjan en la ciencia ficción, pero se pueden introducir de manera fácil supuestos alternativos en las proyecciones.

Existe evidentemente necesidad de aumentar el conocimiento de la situación actual de las ciudades en las naciones subdesarrolladas. No es menos esencial reevaluar la perspectiva, elaborar nuevos enfoques y políticas, tratar de lograr una nueva comprensión, por todos los sectores, de las complejas cuestiones del mañana Las áreas urbanas se beneficiarán del desarrollo global económico y social, pero a su vez, su propio desarrollo saludable puede ser una contribución positiva a la realización de objetivos más amplios.: La perspectiva desalentadora que, por falta de políticas adecuadas, está ahora a la vista es una proliferación de ciudades subdesarrolladas; ciertamente se requiere otra perspectiva. En última instancia, estamos tratando no con terrenos y concreto, o con autopistas, proyectos de vivienda y centros comunitarios, o con agua, parques o atmósferas contaminadas, sino con personas - seres humanos que deben vivir y trabajar juntos, que pueden aspirar a estar solos juntos, y que desafortunadamente tienen la costumbre de multiplicarse (juntos) en medida hasta ahora no prevista.

36 Lowdon Wingo, Jr., "Urban Space in a Policy Perspective", en L. Wingo, Jr., compilador, Cities and Space - The Future Use of Urban Land (Baltimore, The Johns Hopkins Press, 1962), p. 4.

37 Lewis Mumford, The City in History (Nueva York, Harcourt, Brace and World, 1961), p. 527.

38 Departamento del Plan Regulador de Monterrey, N. L., y Municipios Vecinos, El plan director de la subregión Monterrey (Monterrey, Dirección General de Planificación [Gobierno del Estado de Nuevo León], 1967). 